\title{
PR R

\section{Pulse power measurements and attenuator characterization of the hard X-ray beamline at the Linac Coherent Light Source}

Sanghoon Song, Roberto Alonso-Mori, Matthieu Chollet, Yiping Feng, James M. Glownia, et al.

Sanghoon Song, Roberto Alonso-Mori, Matthieu Chollet, Yiping Feng, James M. Glownia, Henrik T. Lemke, Marcin Sikorski, Diling Zhu, Stefan Moeller, Hae Ja Lee, Mark S. Hunter, Gabriella Carini, Kai Tiedtke, Ulf Jastrow, Andrey Sorokin, Mathias Richter, Shigeki Owada, Kensuke Tono, Norio Saito, Takahiro Tanaka, Masahiro Kato, Makina Yabashi, Aymeric Robert, "Pulse power measurements and attenuator characterization of the hard X-ray beamline at the Linac Coherent Light Source," Proc. SPIE 11038, X-Ray FreeElectron Lasers: Advances in Source Development and Instrumentation V, 1103810 (24 April 2019); doi: 10.1117/12.2520827 


\title{
Pulse power measurements and attenuator characterization of the hard X-ray beamline at the Linac Coherent Light Source
}

\author{
Sanghoon Song ${ }^{\mathrm{a}}$, Roberto Alonso-Mori ${ }^{\mathrm{a}}$, Matthieu Chollet ${ }^{\mathrm{a}}$, Yiping Feng ${ }^{\mathrm{a}}$, James M. Glownia ${ }^{\mathrm{a}}$, \\ Henrik T. Lemke ${ }^{\mathrm{a}}$, Marcin Sikorski ${ }^{a}$, Diling Zhu ${ }^{\mathrm{a}}$, Stefan Moeller ${ }^{\mathrm{a}}$, Hae Ja Lee ${ }^{\mathrm{a}}$, Mark S. \\ Hunter $^{\mathrm{a}}$, Gabriella Carini ${ }^{\mathrm{b}}$, Kai Tiedtke ${ }^{\mathrm{c}}$, Ulf Jastrow ${ }^{\mathrm{c}}$, Andrey Sorokin ${ }^{\mathrm{c}}$, Mathias Richter ${ }^{\mathrm{d}}$, \\ Shigeki Owada ${ }^{\mathrm{e}}$, Kensuke Tono ${ }^{\mathrm{e}, \mathrm{f}}$, Norio Saito ${ }^{\mathrm{e}, \mathrm{g}}$, Takahiro Tanaka ${ }^{\mathrm{e}, \mathrm{g}}$, Masahiro Kato ${ }^{\mathrm{e}, \mathrm{g}}$, \\ Makina Yabashie, ${ }^{\mathrm{ef}}$, and Aymeric Robert ${ }^{\mathrm{a}}$ \\ ${ }^{a}$ Linac Coherent Light Source, SLAC National Accelerator Laboratory, 2575 Sand Hill Road, \\ Menlo Park, U.S.A. \\ ${ }^{b}$ Brookhaven National Laboratory, Upton, NY 11973, U.S.A. \\ ${ }^{\mathrm{c}}$ Deutsches Elektronen-Synchrotron, Notkestrasse 85, 22607 Hamburg, Germany \\ ${ }^{d}$ Physikalisch-Technische Bundesanstalt, Abbestraße 2-12, 10587 Berlin, Germany \\ eRIKEN SPring-8 Center, 1-1-1 Kouto, Sayo-cho, Sayo-gun, 679-5198, Hyogo, Japan \\ ${ }^{\mathrm{f} J} J a p a n$ Synchrotron Radiation Research Institute, 1-1-1 Kouto, Sayo-cho, Sayo-gun, 679-5198, \\ Hyogo, Japan \\ ${ }^{g}$ National Institute of Advanced Industrial Science and Technology, NMIJ, 305-8568 Tsukuba, \\ Japan
}

\begin{abstract}
The absolute power of the Linac Coherent Light Source pulses has been measured accurately in the hard X-ray beamline by using simultaneously two detectors: ${ }^{1}$ an X-ray Gas Monitor Detector (XGMD) ${ }^{2}$ in tandem with a radiometer. ${ }^{3}$ From these measurements, we were also able to characterize and calibrate in details our sets of beamline attenuators, in addition to extending an absolute calibration for our beamline intensity monitors. Similarly, we demonstrate that commercial optical power meter has a response in the hard X-ray regime, that can be cross-correlated with the absolute power of the LCLS beam.
\end{abstract}

Keywords: XFEL, Hard X-ray , Intensity monitor, optical laser power meter

\section{INTRODUCTION}

Free electron lasers (FELs) such as the Linac Coherent Light Source (LCLS, Menlo Park, USA) are pulsed sources of X-rays with unprecedented properties: short pulse duration, nearly full transverse coherence but most importantly massive peak power. These properties have already proven to be valuable tools to contribute to investigating problems in many and diverse areas of science. ${ }^{4}$ FEL pulses are typically generated through the self-amplified spontaneous emission (SASE) process, which statistical nature leads to pulse-to-pulse fluctuations in terms of spectrum, intensity, position, and timing. Therefore, beam diagnostics capable of characterizing properties for each shot are crucial for not only accurately analyze experimental results but also guide the experiment while it occurs.

Various beam intensity diagnostics have been developed and evaluated for FEL beams such as fluorescence based gas monitors, ${ }^{5}$ X-ray Gas Monitor Detectors (XGMD) using the photoionization of a gas, ${ }^{2}$ transmissive back-scattering X-ray monitor from thin foils, ${ }^{6,7}$ transmissive thin diamond X-ray monitor, ${ }^{8}$ and calorimeter. ${ }^{3}$ Except for the calorimeter and the XGMD, all other detectors only provide the relative intensity of each X-ray pulses. More recently, some commercial optical laser power meters were evaluated as an absolute X-ray power diagnostics in the soft X-ray regime. ${ }^{9}$

Further author information: Send correspondence to S. Song, e-mail: sanghoon@slac.stanford.edu

X-Ray Free-Electron Lasers: Advances in Source Development and Instrumentation V, edited by Thomas Tschentscher, Luc Patthey, Kai Tiedtke, Marco Zangrando, Proc. of SPIE Vol. 11038, 1103810 @ 2019 SPIE CCC code: $0277-786 X / 19 / \$ 18 \cdot$ doi: $10.1117 / 12.2520827$ 


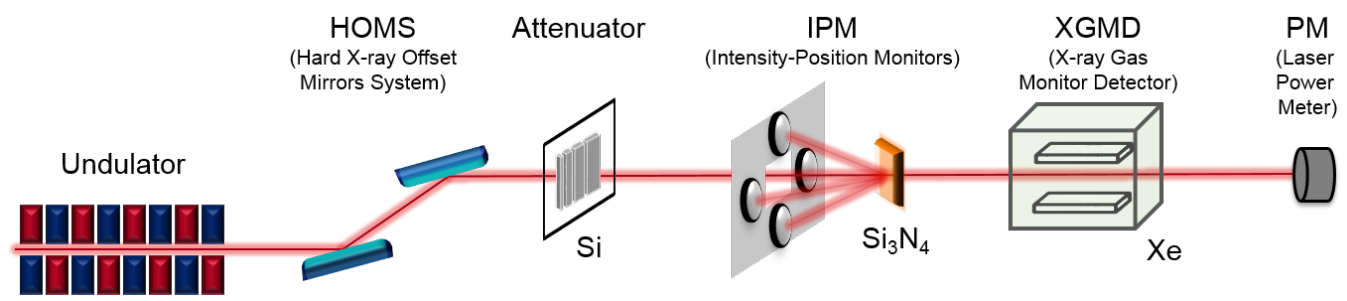

Figure 1. Schematic of the experimental setup at the XPP instrument.

Here we report on the characterization of Intensity-Position Monitors (IPM) and (commercial) laser Power Meter (PM) for providing the absolute pulse power concurrently with measurements with an XGMD in the hard X-ray range of LCLS. ${ }^{1}$ IPMs, which provide a relative power measurement through measuring the X-ray back-scattered from thin foils, are typically permanently installed as an online (i.e., transmissive) monitor at LCLS. Also, four different commercial PMs are evaluated as a potential "portable diagnostics" (i.e., offering the possibility to be placed in any location of interest at the beamline) to provide a measurement of the FEL absolute power in the hard X-ray regime.

\section{EXPERIMENTAL SETUP}

The experiment was performed at the X-ray Pump-Probe (XPP) instrument ${ }^{10}$ at the LCLS. ${ }^{11}$ A schematic of the experimental setup is shown in Fig. 1. The hard X-ray beam is generated from a fixed-gap undulator with the SASE process. It then propagates to the instrument by being first reflected from a set of two Hard X-ray Offset Mirrors (i.e., Hard X-ray Offset Mirror System, HOMS), ${ }^{12,13}$ to suppress higher-order harmonic and bremsstrahlung above $25 \mathrm{keV}$. The XPP instrument solid attenuators consist of a set of ten silicon foils of different thicknesses. These can be inserted individually or as a combination of them, based on the level of attenuation required for a specific X-ray photon energy. At XPP, users specify which transmission level is expected, and the control software will select the nearest transmission reachable by using any combination of foil thicknesses automatically. The Intensity-Position Monitors (IPM) measure a transmissive back-scattered intensity from a thin target by four diodes, which are arranged in quadrants. ${ }^{6,7}$ It provides a measurement of the relative intensity (and position) of individual X-ray pulses. The configuration of the IPM used in our experiment was $0.2 \mu \mathrm{m}$ of Si3N4 as a target and $620 \mathrm{pF}$ gain for the readout electronics.$^{14}$ The X-ray Gas Monitor Detector (XGMD), provided by the Deutsches Elektronen-Synchrotron (DESY, Hamburg, Germany), measures single-shot X-ray absolute power by counting ions of rare gas atoms (i.e., xenon) at a pressure of $\approx 10^{-2} \mathrm{~Pa}^{2}$ The XGMD was cross-calibrated with a radiometer. ${ }^{1}$ Commercial pyroelectric optical laser power meter heads (Coherent: J-10MB-HE) were purchased. These are coated with black paint and have a $10 \mathrm{~mm}$ active area. They are typically expected to function for powers ranging from $10 \mu \mathrm{J}$ up to $20 \mathrm{~mJ}$. Their output is from a power meter controller(Coherent, LabMax-TO). The measurement was performed on four individual heads that have the same manufacture characteristics. The experiment was performed with hard X-rays ranging from $7 \mathrm{keV}$ up to $9.5 \mathrm{keV}$, with a nominal electron charge of $180 \mathrm{pC}, 40 \mathrm{fs}$ pulse length, $120 \mathrm{~Hz}$ repetition rate, and in SASE lasing conditions.

\section{ATTENUATOR CALIBRATION}

In order to characterize each detector over a large range of X-ray power, we used solid attenuators. Each Silicon foil can present a thickness variance as compared to the one provided by the manufacturer. Figure. 2.(a) shows the average X-ray power measured by the XGMD at two different X-ray photon energies, that are normalized with the transmission calculated from the nominal thickness of the foils. For both X-ray photon energies, we observed strong variations as a function of the nominal thickness instead of an expected constant. We, therefore, use the experimental data at various hard X-ray photon energies to retrieving the effective thickness of all foils involved in providing various levels of transmission; thus allowing us to calibrate the individual foil thickness. The results are summarized in table 1 where for $7 \mathrm{keV}$ X-rays the combination of foils is described, the nominal 

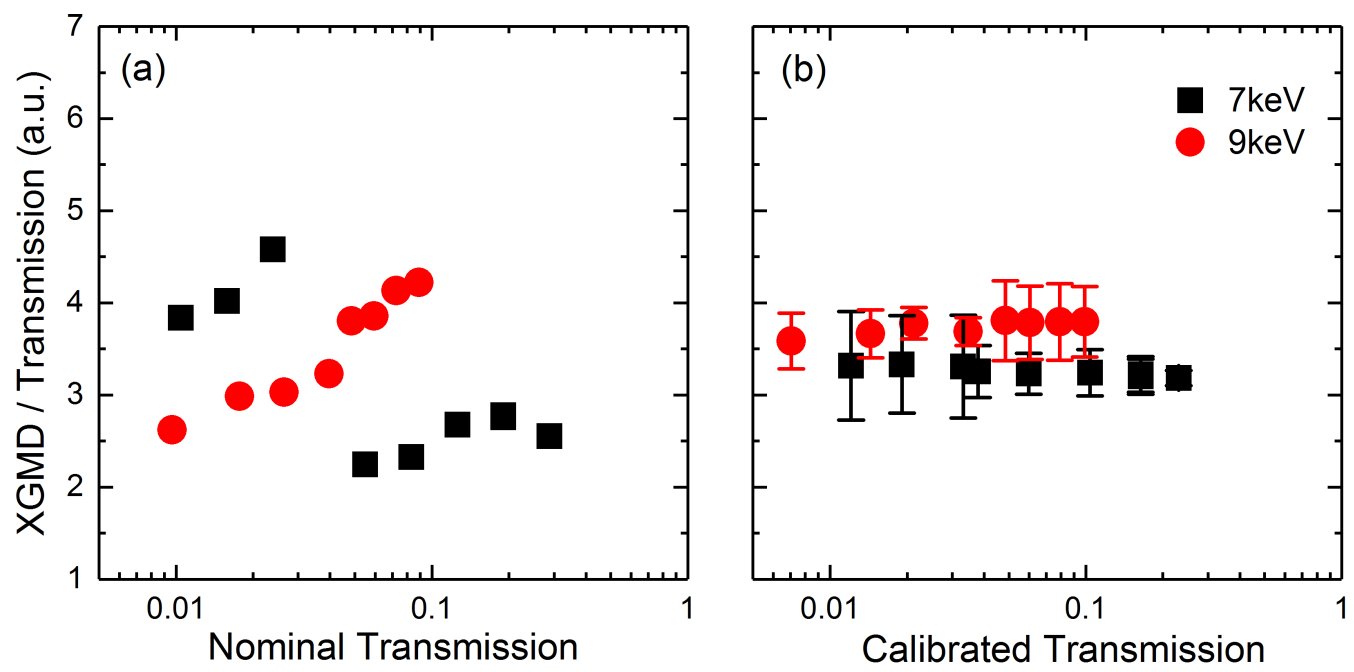

Figure 2. Average XGMD measurements normalized by the transmission using the a) nominal foil thickness and b) calibrated foil thickness

total foil thickness and transmission are provided, and the outcomes of the calibration procedure are provided both in terms of effective transmission and deduced combined thickness. The calibrated transmission are used to generate Figure. 2. (b) that now present a constant behavior as expected within the error bars. By using various combinations of foils, one can then achieve the effective thickness of individual foil as displayed in Table 2. The deviation between nominal and effective thickness ranged from $4 \%$ up to $22 \%$.

Table 1. Foils combinations with various attenuation levels at $E_{\mathrm{X} \text {-ray }}=7 \mathrm{keV}$, Symbol X indicates which individual foils are used for each combination. The transmission and total equivalent Silicon thickness are provided for this X-ray photon energy both from the nominal values but also the calibration procedure.

\begin{tabular}{c|c|c|c|c|c|c|c}
\hline \multicolumn{3}{|c|}{ Foils $(\mu \mathrm{m})$} & \multicolumn{2}{c|}{ Nominal foils } & \multicolumn{2}{c}{ Calibrated foils } \\
\hline 20 & 40 & 80 & 160 & thickness $(\mu \mathrm{m})$ & Transmission $(\%)$ & thickness $(\mu \mathrm{m})$ & Transmission $(\%)$ \\
\hline $\mathrm{X}$ & $\mathrm{X}$ & - & - & 60 & 28.8 & 70.7 & 87.2 \\
- & - & $\mathrm{X}$ & - & 80 & 19.0 & 109.2 & 16.4 \\
$\mathrm{X}$ & - & $\mathrm{X}$ & - & 100 & 12.6 & 135.8 & 10.4 \\
- & $\mathrm{X}$ & $\mathrm{X}$ & - & 120 & 8.3 & 157.9 & 6.0 \\
$\mathrm{X}$ & $\mathrm{X}$ & $\mathrm{X}$ & - & 140 & 5.5 & 164.3 & 3.8 \\
$\mathrm{X}$ & - & - & $\mathrm{X}$ & 180 & 2.4 & 190.9 & 3.3 \\
- & $\mathrm{X}$ & - & $\mathrm{X}$ & 200 & 1.6 & 213.0 & 1.9 \\
$\mathrm{X}$ & $\mathrm{X}$ & - & $\mathrm{X}$ & 220 & 1.0 & 1.2 \\
\hline
\end{tabular}

Table 2. Nominal and calibrated thickness of each individual foils using data from measurements performed at 7, 8, 9, and $9.5 \mathrm{keV}$.

\begin{tabular}{c|c}
\hline Nominal thickness $(\mu \mathrm{m})$ & Calibrated thickness $(\mu \mathrm{m})$ \\
\hline 20 & $22.0 \pm 0.8$ \\
40 & $48.7 \pm 0.4$ \\
80 & $87.2 \pm 2.8$ \\
160 & $142.3 \pm 6.7$ \\
320 & $333.2 \pm 4.0$ \\
\hline
\end{tabular}




\section{IPM CHARACTERIZATION}

In order to characterize the IPM, the measurements were performed from 7 to $9.5 \mathrm{keV}$ with various attenuation levels. Figure 3.(a) shows the correlation of the measurement at $9 \mathrm{keV}$ from the IPM as a function of the XGMD signal. The XGMD data are corrected to reflect the same level of power at the IPM position (i.e., correcting by the X-ray transmission of all components located between the IPM and XGMD). This was performed in "pink beam", thus using the full spectrum of the first harmonic. The solid line shows a fit to the data showing appreciable linearity of response of both detectors. The results of the linear fit for all energies are provided for converting from IPM output to pulse power $(\mu \mathrm{J})$ in Table 3. Figure. 3.(b) shows the relative linearity of both detectors (i.e., IPM signal converted from Volts to power and then normalized by the value of XGMD) as a function of the XGMD signal. In order to characterize quantitatively these results, Fig. 3.(c) provided the standard deviation $\sigma$ (i.e., as obtained from a Gaussian fit of the data distribution on intervals of XGMD powers) of the data presented in Fig. 3. (b). It indicates that the degree of linearity (i.e., inversely proportional to $\sigma$ ) increases as a function of the signal strength and reaches levels better than $4 \%$ for powers larger than $40 \mu \mathrm{J}$.
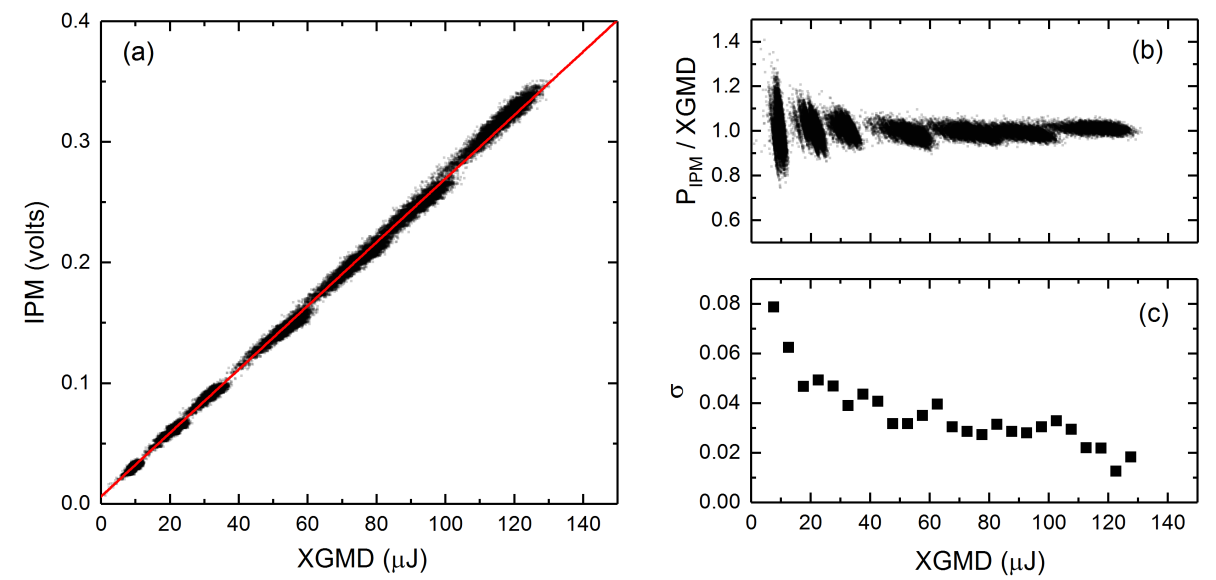

Figure 3. a) Correlation between the pulse power measured by the XGMD and output of an IPM at $E_{\mathrm{X} \text {-ray }}=9 \mathrm{keV}$. The solid line indicates the fit to the data using a linear function. b) Relative linearity of IPM and XGMD, the IPM output measurement convert from volts to pulse power, and then normalize it by the pulse power from the XGMD. c) Dependence of the standard deviation $\sigma$ of the data in (b) as a function of the pulse power measured by the XGMD.

Table 3. Summary of linear fitting parameters to convert from IPM output (Volts) to pulse power $(\mu \mathrm{J})$ for X-ray photon energies from $7 \mathrm{keV}$ up to $9.5 \mathrm{keV}$.

\begin{tabular}{c|c|c}
\hline$E_{\text {X-ray }}(\mathrm{keV})$ & Slope & Intercept \\
\hline 7 & 261.9 & -12.3 \\
8 & 278.9 & -1.9 \\
9 & 378.2 & -2.1 \\
9.5 & 431.1 & 0.75 \\
\hline
\end{tabular}

\section{CHARACTERIZATION OF COMMERCIAL LASER POWER METERS}

Measurements with commercial laser power meters (PM) were performed for X-ray photon energies from $7 \mathrm{keV}$ to $9.5 \mathrm{keV}$ with various attenuation levels. PM signal subtracted a background noise and then corrected for the contribution of absorption of optical components between the XGMD and the PM. These corrections did consider the presence of the third harmonic in the incident beam. The harmonic ratio between the third and first harmonic in the full incident beam has been measured to be below $0.5 \%$ in this X-ray photon energy range as described in Reference. ${ }^{1}$ The range over which all PM heads and its controller did not present any saturation was below output values of $300 \mu \mathrm{J}$. This configuration corresponds to an incident pulse of power below $100 \mu \mathrm{J}$ as measured by the XGMD. 

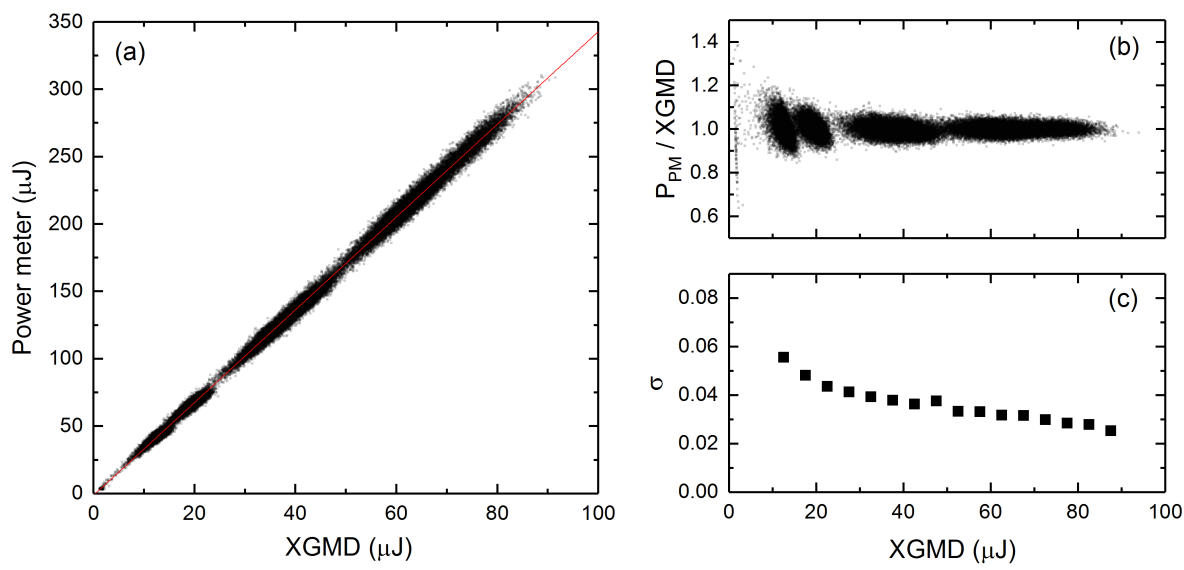

Figure 4. a) Correlation between the XGMD and laser power meter (PM1) outputs at $E_{\mathrm{X} \text {-ray }}=7 \mathrm{keV}$. The solid red line indicates the fit to the data using a linear function. b) Linearity of laser power meter (PM1) and the XGMD, the normalized pulse power of power meter $\left(\mathrm{P}_{\mathrm{PM} 1}\right)$ by the XGMD and the XGMD. c) Dependence of the standard deviation $\sigma$ of the data in (b) as a function of the pulse power measured by the XGMD.

Figure 4. (a) presents the correlation between the PM1 and XGMD signals at $E_{\mathrm{X} \text {-ray }}=7 \mathrm{keV}$. The solid line is a linear fit to the data. The results of the fit are provided in Table. 4 for this X-ray photon energy but also for 8 , and $9.5 \mathrm{keV}$. The table also presents the results when measuring with different power meter heads (i.e., PM2, 3, and 4). For a given X-ray photon energy we do observe that the three Power meter heads 1,2 , and 3 present very similar responses. However, PM4 shows a difference response of nearly $15 \%$ as compared to the other power meter heads. Whereas there is no firm explanation of this discrepancy, these results indicate that some of the PM heads can slightly change in terms of their performance, as a possible result of changes in their manufacturing processes. It most importantly informs that each power meter head should be properly calibrated against a calibrated detector such as the XGMD before being used as an absolute power diagnostic. Similarly to the analysis performed with the IPM which results are displayed in Fig. 3, Fig. 4 presents the results of such analysis for the power meter head PM1. Once again this diagnostics presents good linearity, which for incident signals larger than $30 \mu \mathrm{J}$ ( i.e., as measured by the XGMD) provides a degree of linearity better than $4 \%$

Table 4. Summary of linear fit parameters to convert from output of four different laser power meter $(\mu \mathrm{J})$ to X-ray pulse energy $(\mu \mathrm{J})$ from $7 \mathrm{keV}$ to $9.5 \mathrm{keV}$.

\begin{tabular}{c|c|c|c|c|c|c|c|c}
$E_{\text {X-ray }}$ & \multicolumn{2}{|c|}{ PM1 } & \multicolumn{2}{c|}{ PM2 } & \multicolumn{2}{c|}{ PM3 } & \multicolumn{2}{c}{ PM4 } \\
\cline { 2 - 9 }$(\mathrm{keV})$ & Slope & Intercept & Slope & Intercept & Slope & Intercept & Slope & Intercept \\
\hline 7 & 0.29 & 0.40 & - & - & - & - & & \\
8 & 0.28 & -1.74 & 0.28 & -1.32 & 0.27 & -1.32 & 0.22 & -1.18 \\
9.5 & 0.30 & -4.94 & 0.27 & -0.01 & 0.25 & 0.40 & 0.20 & 0.88 \\
\hline
\end{tabular}

\section{SUMMARY}

We characterized the performance of one of the XPP instrument diagnostics (IPM) which is permanently installed at the beamline and is used in a transmissive way. We most importantly were able to derive a conversion of its signal in units of absolute power. These will help to provide an estimate of the absolute incident power for all $\mathrm{XPP}$ experiments within this X-ray photon energy range and experimental conditions. We also demonstrated the feasibility of using a commercial laser power meter as an absolute diagnostic for measuring the power of the beam. These provide the advantage that they can be used at various locations along the experimental setup; i.e., it is a portable diagnostic. However, each power meter head needs to be properly calibrated with X-rays against another detector such as the XGMD. 


\section{ACKNOWLEDGMENTS}

Use of the Linac Coherent Light Source (LCLS), SLAC National Accelerator Laboratory, is supported by the U.S. Department of Energy, Office of Science, Office of Basic Energy Sciences under Contract No. DE-AC0276SF00515. The authors want to thank the technical support provided by RIKEN member Toshiyuki Murakami.

\section{REFERENCES}

[1] Song, S., Alonso-Mori, R., Chollet, M., Feng, Y., Glownia, J. M., Lemke, H. T., Sikorski, M., Zhu, D., Moeller, S., Lee, H.-J., Hunter, M. S., Carini, G., Tiedtke, K., Jastrow, U., Sorokin, A., Richter, M., Owada, S., Tono, K., Saito, N., Tanaka, T., Kato, M., Yabashi, M., and Robert, A., "Measurement of the absolute number of photons of the hard x-ray beamline at the linac coherent light source," Journal of Synchrotron Radiation 26(2), 320-327 (2019).

[2] Tiedtke, K., Sorokin, A. A., Jastrow, U., Juranić, P., Kreis, S., Gerken, N., Richter, M., Arp, U., Feng, Y., Nordlund, D., Soufli, R., Fernndez-Perea, M., Juha, L., Heimann, P., Nagler, B., Lee, H. J., Mack, S., Cammarata, M., Krupin, O., Messerschmidt, M., Holmes, M., Rowen, M., Schlotter, W., Moeller, S., and Turner, J. J., "Absolute pulse energy measurements of soft x-rays at the linac coherent light source," Optics Express 22(18), 21214-21226 (2014).

[3] Tanaka, T., Kato, M., Saito, N., Tono, K., Yabashi, M., and Ishikawa, T., "Room-temperature calorimeter for x-ray free-electron lasers," Review of Scientific Instruments 86(9), 093104 (2015).

[4] Bostedt, C., Boutet, S., Fritz, D. M., Huang, Z., Lee, H. J., Lemke, H. T., Robert, A., Schlotter, W. F., Turner, J. J., and Williams, G. J., "Linac coherent light source: The first five years," Reviews of Modern Physics 88(1), 015007 (2016).

[5] Hau-Riege, S. P., Bionta, R. M., Ryutov, D. D., London, R. A., Ables, E., Kishiyama, K. I., Shen, S., McKernan, M. A., McMahon, D. H., Messerschmidt, M., Krzywinski, J., Stefan, P., Turner, J., and Ziaja, B., "Near-ultraviolet luminescence of $\mathrm{n} 2$ irradiated by short x-ray pulses," Physical Review Letters 105(4), 043003 (2010).

[6] Feng, Y., Feldkamp, J. M., Fritz, D., Cammarata, M., Aymeric, R., Caronna, C., Lemke, H. T., Zhu, D., Lee, S., Boutet, S., Garth, W., Kensuke, T., Makina, Y., and Jerome, B. H., "A single-shot intensity-position monitor for hard x-ray fel sources," Proc. SPIE 8140, 81400Q (2011).

[7] Tono, K., Kudo, T., Yabashi, M., Tachibana, T., Feng, Y., Fritz, D., Hastings, J., and Ishikawa, T., "Singleshot beam-position monitor for x-ray free electron laser," Review of Scientific Instruments 82(2), 023108 (2011).

[8] Roth, T., Freund, W., Boesenberg, U., Carini, G., Song, S., Lefeuvre, G., Goikhman, A., Fischer, M., Schreck, M., Grünert, J., and Madsen, A., "Pulse-resolved intensity measurements at a hard x-ray fel using semi-transparent diamond detectors," Journal of synchrotron radiation 25(1), 177-188 (2018).

[9] Heimann, P., Moeller, S., Carbajo, S., Song, S., Dakovski, G., Nordlund, D., and Fritz, D., "Laser power meters as an x-ray power diagnostic for lcls-ii," Journal of synchrotron radiation 25(1), 72-76 (2018).

[10] Chollet, M., Alonso-Mori, R., Cammarata, M., Damiani, D., Defever, J., Delor, J. T., Feng, Y., Glownia, J. M., Langton, J. B., Nelson, S., Ramsey, K., Robert, A., Sikorski, M., Song, S., Stefanescu, D., Srinivasan, V., Zhu, D., Lemke, H. K., and D., F., "The x-ray pump-probe instrument at the linac coherent light source," Journal of Synchrotron Radiation 22(3), 503-507 (2015).

[11] White, W. E., Robert, A., and Dunne, M., "The linac coherent light source," Journal of Synchrotron Radiation 22(3), 472-476 (2015).

[12] McCarville, T. J., Stefan, P. M., Woods, B., Bionta, R. M., Soufli, R., and Pivovaroff, M. J., "Optomechanical design considerations for the linac coherent light source x-ray mirror system," Proc. SPIE 7077, $70770 \mathrm{E}(2008)$.

[13] Soufli, R., Pivovaroff, M. J., Baker, S. L., Robinson, J. C., Gullikson, E. M., Mccarville, T., Stefan, P., Aquila, A., Ayers, J., McKernan, M. A., and Bionta, R. M., "Development, characterization and experimental performance of x-ray optics for the lcls free-electron laser," Proc. SPIE 7077, 707716 (2008).

[14] Herrmann, S., Hart, P., Freytag, M., Pines, J., Weaver, M., Sapozhnikov, L., Nelson, S., Koglin, J., Carini, G., Tomada, A., and Haller, G., "Diode readout electronics for beam intensity and position monitors for fels," Journal of Physics: Conference Series 493(1), 012014 (2014). 